\title{
Linear Landau damping and wave energy dissipation in the interstellar medium
}

\author{
I. Lerche $^{1}$ and R. Schlickeiser ${ }^{2}$ \\ 1 Department of Geological Sciences, University of South Carolina, Columbia, SC 29208, USA \\ e-mail: black@geol.sc.edu \\ 2 Institut für Theoretische Physik, Lehrstuhl IV: Weltraum- und Astrophysik, Ruhr-Universität Bochum, \\ 44780 Bochum, Germany \\ e-mail: r.schlickeiser@tp4.ruhr-uni-bochum.de
}

Received 25 July 2000 / Accepted 8 November 2000

\begin{abstract}
The heating rate of the diffuse interstellar medium is calculated from collisionless dissipation of magnetohydrodynamic plasma waves by linear Landau damping. We demonstrate that, when considering individual plasma wave types, two factors are of importance. First, the approximations and conditions under which the wave damping rates are produced must be honored to ensure that one does not step outside the domain of validity of the approximation. Second, when integrating over a wave spectrum due care and diligence must be given in performing the integrals as accurately as possible and not approximating too early in the development. Failure to take such care can lead to inappropriate wave energy loss rates which do not reflect correctly the exact expressions. The numerical estimates produced for linear Landau damping indicate that there is little, if any, disparity between radiative cooling rates and wave energy loss rates for the fluctiferous ( $\mathrm{H}$ II-regions) and the diffuse interstellar medium when the anisotropy in the wave power spectrum is properly accounted for. Our results show that the interstellar turbulence mostly consists of obliquely propagating compressive fast magnetosonic waves. However, the power spectrum of these waves is not isotropic in wavenumber space but has to be very anisotropic, either elongated mainly along or perpendicular to the ordered magnetic field component.
\end{abstract}

Key words. magnetohydrodynamics (MHD) - plasmas - turbulence - waves - ISM: general - ISM: magnetic fields

\section{Introduction}

According to current understanding (Field et al. 1969; McKee \& Ostriker 1977; McCray \& Snow 1979; Ferriere et al. 1988) the interstellar medium consists of at least three distint phases in approximate pressure equilibrium: cold clouds, warm intercloud medium, and hot coronal gas generated by supernova explosions (Cox \& Smith 1974). Spitzer (1956) originally postulated the existence of hot (3 $10^{5} \mathrm{~K} \leq T \leq 10^{6} \mathrm{~K}$ ) coronal gas which was later confirmed by observations of ultraviolet absorption lines (Jenkins 1978; Hartquist \& Snijders 1982) and studies of the diffuse soft X-ray background (McCammon \& Sanders 1990). $21 \mathrm{~cm}$ radio studies indicate that the warm medium has a temperature between 6000 and $10^{4} \mathrm{~K}$ and a mean HI-density of about $0.8 \mathrm{~cm}^{-3}$, but is very stuctured in colder clouds and a warm intercloud medium of density $0.1-0.2 \mathrm{~cm}^{-3}$. Under such conditions atomic and metallic radiative transitions efficiently cool the gas, so that an ef-

Send offprint requests to: $\mathrm{R}$. Schlickeiser ficient heating mechanism is required in order to maintain the gas temperature.

Various heating mechanisms have been suggested, including background ultraviolet and X-ray heating (e.g. Cheng \& Bruhweiler 1990), cosmic ray heating (e.g. Lerche \& Schlickeiser 1982), and photoelectric emission from grains. Here we investigate the heating of the warm gas by the collisionless damping of interstellar magnetohydrodynamic waves. The collisionless dissipation of various kinds of waves in the interstellar medium has had a long history (Barnes 1966; Kulsrud \& Pearce 1969; McIvor 1977; Cesarsky 1980; Ferriere et al. 1988; Spangler 1991; Minter \& Spangler 1997). Underlying motivations for this continued interest are to obtain a detailed understanding of the mechanisms responsible for the heating and/or cooling of the interstellar medium (see the review in Spangler 1991), and to understand how a balance is achieved or maintained between the competing processes.

In a theoretical description the heating $(\epsilon)$ and cooling $(\lambda)$ rates enter the heat conduction equation of a viscous 
fluid (Landau \& Lifschitz 1981)

$$
\begin{array}{r}
\rho T\left[\frac{\partial s}{\partial t}+\boldsymbol{v} \cdot \nabla s\right]=\operatorname{div}(\kappa \nabla T)+\sigma_{i k} \frac{\partial v_{i}}{\partial x_{k}} \\
+\epsilon(\rho, T)-\lambda(\rho, T)
\end{array}
$$

where $s$ denotes the fluid entropy, $\rho$ and $\boldsymbol{v}$ the fluid density and velocity, $\kappa$ is the heat conduction coefficient and $\sigma_{i k}$ the viscous stress tensor. Formally, Eq. (1) can be regarded as the fifth equation - besides the continuity equation and the three Euler equations - of the hydrodynamical description of a viscous fluid. Here we consider a stationary interstellar medium with large spatial scales and without strong spatial inhomogenities, i.e. far away from phase boundaries and shock waves, so that to a first approximation we can neglect all terms in Eq. (1) with spatial and time derivatives. In this case Eq. (1) reduces to the simple balance of heating and cooling rates

$\epsilon(\rho, T)=\lambda(\rho, T)$.

Our discussion of the heating by collisionless Landau damping will be based in Eq. (2), and thus is valid only in the stationary and homogenous interstellar medium.

\section{Magnetic field fluctuation spectrum in the Galaxy}

In some of the earlier works concerned with these wave dissipation processes, there was, at that time, extremely limited information on the power spectrum of waves in the interstellar medium. Limited attempts had been made by Jokipii \& Lerche (1969) and Jokipii et al. (1969), using Faraday rotation and pulsar signal dispersion to obtain rough estimates of the wave fluctuation strength. But the quantity and quality of data at that time led to a very incomplete knowledge of wave spectra. In turn, this uncertainty bled through to large uncertainties on the wave dissipation rates.

Over the last thirty years or so, due to continued refinement in observational techniques, there has been substantial progress in the understanding of wave spectra, directly related to the massive improvements in quality and quantity of data (see e.g. Rickett 1990). At the present time of writing, a synthesis of available data would seem to indicate that a power spectrum of waves in the form

$P(\boldsymbol{k})=C_{\mathrm{B}}^{2}\left[k_{\|}^{2}+\Lambda k_{\perp}^{2}\right]^{-(2+s) / 2}$

can account for the observations (Spangler 1991). Here $k_{\|}$ $\left(k_{\perp}\right)$ is the wavenumber parallel (perpendicular) to the ambient magnetic field; $s$ is the spectral index which is estimated to lie between about $3 / 2$ and $5 / 3 ; \Lambda$ is the anisotropy parameter. Isotropy occurs if $\Lambda=1$, whereas if the wave turbulence is more along the lines of thin "platelets" paralleling the ambient field, as suggested by Goldreich \& Sridhar (1995), then $\Lambda \gg 1$. The constant $C_{\mathrm{B}}^{2}$ is such that

$\int \mathrm{d}^{3} k P(\boldsymbol{k})=(\delta B)^{2}$ where $\delta B$ denotes the total fluctuating magnetic field component. The form of the wave spectrum given by Eq. (3) is taken to operate only between some small wavenumber, $k=k_{\min }$, and a large wavenumber, $k=k_{\max }$, with $k=|\boldsymbol{k}|=\left[k_{\|}^{2}+k_{\perp}^{2}\right]^{1 / 2}$. Spangler (1991) suggests that these wavenumbers are related to outer and inner scale lengths, $l_{\min }$ and $l_{\max }$, respectively with $l_{\min }=2 \pi / k_{\max }$, $l_{\max }=2 \pi / k_{\min }$. The physical bounds of $l_{\min }$ and $l_{\max }$ are not precisely known, but probably related to the Whistler wave resonance limit of the interstellar electrons and the physical size of the warm intercloud medium i.e. the mean cloud distance, respectively. The inner scale is estimated by Spangler (1991) to be of the general order of $l_{\min }=10^{7} l_{7} \mathrm{~cm},\left(l_{7}=1\right)$, with the outer scale of order $l_{\max }=10^{17} L_{17} \mathrm{~cm}$ with $L_{17}=1$ in hot, ionized regions, and $L_{17}=30$ in the diffuse phase of the interstellar medium. In our study here we assume that the power spectrum (3) holds in the stationary and homogenous warm intercloud medium.

The power spectrum itself results from the balance of all wave damping and driving processes, although a detailed theory and explanation currently is not available. Therefore our calculation will be limited in the sense that we assume a given and fixed power spectrum to determine the heating rate of the interstellar medium, but we do not self-consistently investigate the effect of this energy loss rate on the form of the power spectrum

In any event, it would seem that a sufficiently detailed wave power spectrum is now available, based on a synthesis of modern observations and theoretical improvements, that a sharper determination of wave dissipation rates can be given. The purpose of this paper is to re-examine the wave dissipation rate for linear Landau damping. In other communications, the non-linear Landau damping mechanism will also be re-examined for it, too, has some interesting properties.

\section{Energy loss rate}

For waves damping at a rate $\gamma(\boldsymbol{k})$, the energy loss rate $\epsilon$ is conventionally written in the form (Spangler 1991)

$\epsilon=\frac{1}{4 \pi} \int \mathrm{d}^{3} k P(\boldsymbol{k}) 2 \gamma(\boldsymbol{k})$.

\subsection{Oblique wave reduction}

For an obliquely propagating magnetosonic wave the damping rate is given by Ginzburg (1961, p. 218, Eq. (14.56)) as

$$
\gamma=\left(\frac{\pi}{8}\right)^{1 / 2} \frac{\sin ^{2} \theta}{\cos \theta} k v_{\mathrm{i}}\left[v_{\mathrm{i}} / v_{\mathrm{e}}+5 \exp \left[-V_{\mathrm{A}}^{2} /\left(2 v_{\mathrm{i}}^{2} \cos ^{2} \theta\right)\right]\right]
$$

where $\sin \theta=\left|k_{\perp}\right| / k, V_{\mathrm{A}}$ is the Alfven speed, $v_{\mathrm{i}}\left(v_{\mathrm{e}}\right)$ is the ion (electron) thermal speed. Conditions are also attached to the domain of validity of Eq. (6). These conditions, as 
spelled out by Ginzburg (1961), are:

(1) Both ions and electrons are taken to be at the same temperature and both are described by isotropic Maxwellian distributions, $v_{\mathrm{e}}=\sqrt{k_{\mathrm{B}} T / m_{\mathrm{e}}}, v_{\mathrm{i}}=\sqrt{k_{\mathrm{B}} T / m_{\mathrm{i}}}=$ $v_{\mathrm{e}}\left(m_{\mathrm{e}} / m_{\mathrm{i}}\right)^{1 / 2}$

(2) One must have $V_{\mathrm{A}} \gg v_{\mathrm{i}}$ and $v_{\mathrm{e}} \gg v_{\mathrm{i}}$;

(3) Eq. (6) is valid only in the angular range described through

$v_{\mathrm{e}}|\cos \theta| \gg\left[V_{\mathrm{A}}^{2}+3 v_{\mathrm{i}}^{2} \sin ^{2} \theta\right]^{1 / 2} \gg v_{\mathrm{i}}|\cos \theta|$.

The left part of inequality (7) provides the restriction

$|\cos \theta| \geq \mu_{\mathrm{L}} \equiv\left[\frac{V_{\mathrm{A}}^{2}+3 v_{\mathrm{i}}^{2}}{v_{\mathrm{e}}^{2}+3 v_{\mathrm{i}}^{2}}\right]^{1 / 2}$.

In general, $\mu_{\mathrm{L}} \simeq V_{\mathrm{A}} / v_{\mathrm{e}} \ll 1$ for the cases of interest later in this paper. The right part of inequality (7) is always satisfied when condition 2 is in force.

The exponential factor $\exp \left[-V_{\mathrm{A}}^{2} /\left(2 v_{\mathrm{i}}^{2} \cos ^{2} \theta\right)\right]$ varies between $\exp \left[-V_{\mathrm{A}}^{2} /\left(2 v_{\mathrm{i}}^{2}\right)\right]$ and $\exp \left[-v_{\mathrm{e}}^{2} /\left(2 v_{\mathrm{i}}^{2}\right)\right]$ as $|\cos \theta|$ traverses $1 \geq|\cos \theta| \geq \mu_{\mathrm{L}}$. Because both of these exponential factors are exceedingly small one can write, to a very accurate approximation, that

$\gamma \simeq\left(\frac{\pi}{8}\right)^{1 / 2} \frac{\sin ^{2} \theta}{\cos \theta} k v_{\mathrm{i}}^{2} v_{\mathrm{e}}^{-1}$

As mentioned, Eqs. (6) and (9) are valid for isotropic Maxwellians with $T_{\mathrm{e}}=T_{\mathrm{i}}$. This assumption is adopted here because we want to evaluate the heating rate with the same input parameters as Spangler 1991) to allow the direct comparison of our calculation with his. In future work we want to consider collisionless heating rates also for an interstellar medium in NLTE conditions as X-ray absorption studies lead to $T_{\mathrm{e}}>T_{\mathrm{i}}$ and ordered magnetic fields may enforce anisotropic temperatures $\left(T_{\|} \neq T_{\perp}\right)$. Both cases require the determination of appropriate, more general damping rates in these changed plasma conditions.

With the damping rate (9) the energy loss rate can then be written

$\epsilon=\frac{C_{\mathrm{B}}^{2} v_{\mathrm{i}}^{2}}{2^{5 / 2} \pi^{1 / 2} v_{\mathrm{e}}} \int \mathrm{d}^{3} k \frac{k^{-(1+s)} \sin ^{2} \theta}{\cos \theta\left[\cos ^{2} \theta+\Lambda \sin ^{2} \theta\right]^{1+(s / 2)}}$,

where the integral range is restricted to be in $1 \geq|\cos \theta| \geq$ $\mu_{\mathrm{L}}$ and $k_{\min } \leq k \leq k_{\max }$. With spherical coordinates for $\boldsymbol{k}$ one can simplify Eq. (10) for general $s$ to

$\epsilon=\left(\frac{\pi}{2}\right)^{1 / 2} C_{\mathrm{B}}^{2} v_{\mathrm{i}}^{2} v_{\mathrm{e}}^{-1} \frac{k_{\max }^{2-s}-k_{\min }^{2-s}}{2-s} I\left(\mu_{\mathrm{L}}, \Lambda, s\right)$

with the integral

$I\left(\mu_{\mathrm{L}}, \Lambda, s\right) \equiv \int_{\mu_{\mathrm{L}}}^{1} \mathrm{~d} \mu \frac{\left(1-\mu^{2}\right)}{\mu\left[\mu^{2}+\Lambda\left(1-\mu^{2}\right)\right]^{1+(s / 2)}}$.

For the specific value $s=2$ Eq. (11) becomes

$$
\begin{aligned}
\epsilon(s= & 2)=\left(\frac{\pi}{2}\right)^{1 / 2} C_{\mathrm{B}}^{2} v_{\mathrm{i}}^{2} v_{\mathrm{e}}^{-1} \ln \left(k_{\max } / k_{\min }\right) \\
& \times \int_{\mu_{\mathrm{L}}}^{1} \mathrm{~d} \mu \frac{\left(1-\mu^{2}\right)}{\mu\left[\mu^{2}+\Lambda\left(1-\mu^{2}\right)\right]^{2}} .
\end{aligned}
$$

Note that the lower limit of the $\mu$-integral has been set to $\mu=\mu_{\mathrm{L}}$ whereas the Ginzburg (1961) damping formula is really valid in $\mu \gg \mu_{\mathrm{L}}$. Thus Eqs. (11) and (13) provide overestimates of the energy loss rate.

For completeness we note that the integral (12) can be formally expressed in terms of hypergeometric functions,

$$
\begin{aligned}
& I\left(\mu_{\mathrm{L}}, \Lambda, s\right)=\frac{1}{2+s}\left[\left[\Lambda+(1-\Lambda) \mu_{\mathrm{L}}^{2}\right)\right]^{-(2+s) / 2} \\
& \times{ }_{2} F_{1}\left(1,1+\frac{s}{2} ; 2+\frac{s}{2} ; \frac{\Lambda}{\Lambda+(1-\Lambda) \mu_{\mathrm{L}}^{2}}\right)
\end{aligned}
$$$$
\left.-{ }_{2} F_{1}\left(1,1+\frac{s}{2} ; 2+\frac{s}{2} ; \Lambda\right)\right]
$$

$+\frac{1}{s(1-\Lambda)}\left[1-\left[\Lambda+(1-\Lambda) \mu_{\mathrm{L}}^{2}\right]^{-s / 2}\right]$.

Moreover

$$
\begin{aligned}
& { }_{2} F_{1}\left(1,1+\frac{s}{2} ; 2+\frac{s}{2} ; \frac{\Lambda}{\Lambda+(1-\Lambda) \mu_{\mathrm{L}}^{2}}\right) \\
& =\frac{2+s}{2} \sum_{n=0}^{\infty} \frac{\Gamma\left(n+1+\frac{s}{2}\right)}{\Gamma\left(1+\frac{s}{2}\right) n !}\left[\psi(n+1)-\psi\left(n+1+\frac{s}{2}\right)\right. \\
& \left.\quad-\ln \left(\frac{(1-\Lambda) \mu_{\mathrm{L}}^{2}}{\Lambda+(1-\Lambda) \mu_{\mathrm{L}}^{2}}\right)\right]\left[\frac{(1-\Lambda) \mu_{\mathrm{L}}^{2}}{\Lambda+(1-\Lambda) \mu_{\mathrm{L}}^{2}}\right]^{n}
\end{aligned}
$$

where $\psi(m)$ denotes the digamma function. In order to find the asymptotic behaviour of the integral (12) for different values of $\Lambda$, we will either use Eq. (14) or start directly from the integral expression (12), depending on convenience.

\subsection{Fluctuating magnetic field}

Combining Eqs. (3) and (4) we derive

$(\delta B)^{2}=4 \pi C_{\mathrm{B}}^{2} \frac{k_{\min }^{1-s}}{s-1}\left[1-\left(k_{\min } / k_{\max }\right)^{s-1}\right] J(\Lambda, s)$

where $J(\Lambda=1, s)=1$ and for $\Lambda \neq 1$

$J(\Lambda)=\int_{0}^{1} \mathrm{~d} \mu\left[\mu^{2}+\Lambda\left(1-\mu^{2}\right)\right]^{-(2+s) / 2}$

$=\frac{1}{2 \Lambda^{(2+s) / 2}} \int_{0}^{1} \mathrm{~d} x \frac{1}{x^{1 / 2}[1+((1-\Lambda) x / \Lambda)]^{(2+s) / 2}}$

$=\frac{1}{\Lambda^{(2+s) / 2}}{ }_{2} F_{1}\left(1+\frac{s}{2}, \frac{1}{2} ; \frac{3}{2} ; 1-\Lambda^{-1}\right)$.

Using the transformation formula

${ }_{2} F_{1}(a, b ; c ; z)=(1-z)^{-a}{ }_{2} F_{1}\left(a, c-b ; c ; \frac{z}{z-1}\right)$

readily yields

$J(\Lambda)={ }_{2} F_{1}\left(1+\frac{s}{2}, 1 ; \frac{3}{2} ; 1-\Lambda\right)$. 


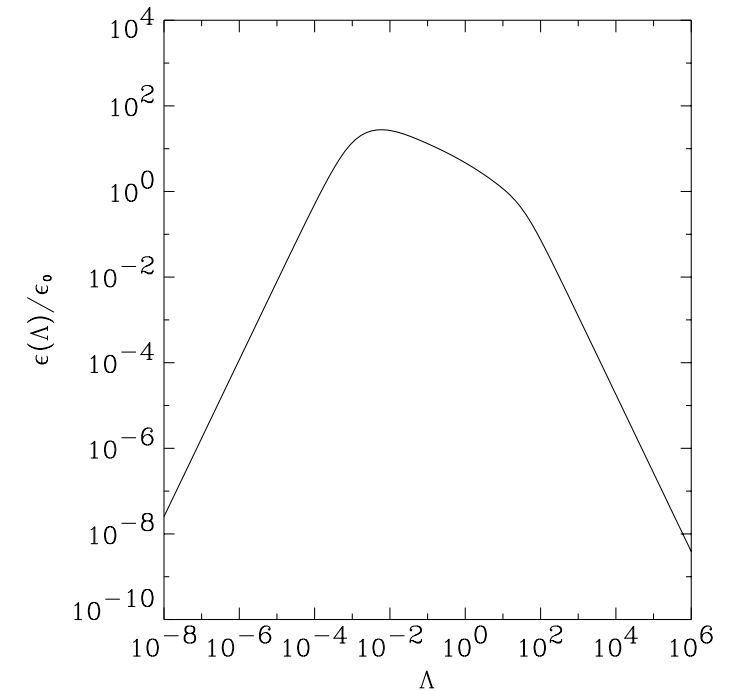

Fig. 1. Variation of the energy loss rate as a function of the anisotropy parameter $\Lambda$ for a turbulence spectral index $s=5 / 3$ and $\mu_{\mathrm{L}}=0.05$

\subsection{Final energy loss rate}

Solving Eq. (16) for $C_{\mathrm{B}}^{2}$ and inserting the result in Eq. (11) we obtain the final expression for the energy loss rate

$\epsilon(\Lambda)=\epsilon_{0} \frac{I\left(\mu_{\mathrm{L}}, \Lambda, s\right)}{J(\Lambda, s)}$

with

$$
\begin{aligned}
\epsilon_{0}=2^{-5 / 2} \pi^{-1 / 2} \frac{s-1}{2-s} \frac{\left[1-\left(k_{\min } / k_{\max }\right)^{2-s}\right]}{\left[1-\left(k_{\min } / k_{\max }\right)^{s-1}\right]} \\
\times k_{\max }^{2-s} k_{\min }^{s-1}(\delta B)^{2} \frac{v_{\mathrm{i}}^{2}}{v_{\mathrm{e}}} .
\end{aligned}
$$

In Fig. 1 we have numerically calculated the variation of the energy loss rate as a function of the anisotropy parameter $\Lambda$ for the turbulence spectral index $s=5 / 3$ and $\mu_{\mathrm{L}} \simeq V_{\mathrm{A}} / v_{\mathrm{e}}=0.05$ (see also Sect. 5.1). It can be seen that in this case the energy loss rate attains its maximum at values of about $\Lambda \simeq 10^{-2}$ and that it varies with different functional dependences at small or large values of $\Lambda$. In the following we derive analytically the asymptotic behaviours of the two integrals $I\left(\mu_{\mathrm{L}}, \Lambda, s\right)$ and $J(\Lambda, s)$ for different values of the anisotropy parameter $\Lambda$ and the turbulence spectral index $s$. The obtained asymptotic baviours - summarized in Table 1 - are in full agreement with the numerical results of Fig. 1.

\section{Different limits of the anisotropy parameter $\Lambda$}

\subsection{Isotropic turbulence $(\Lambda=1)$}

For an isotropic spectrum of waves one has $J(\Lambda=1, s)=1$ and

$I\left(\mu_{\mathrm{L}}, \Lambda=1, s\right)=\ln \left(\frac{1}{\mu_{\mathrm{L}}}\right)-\frac{1}{2}\left(1-\mu_{\mathrm{L}}^{2}\right)$ so that with $\mu_{\mathrm{L}} \simeq V_{\mathrm{A}} / v_{\mathrm{e}} \ll 1$ Eq. (20) becomes

$\epsilon_{\text {isotropic }}=\epsilon_{0}\left[\ln \left(v_{\mathrm{e}} / V_{\mathrm{A}}\right)-\frac{1}{2}\right]$.

In terms of the inner and outer scale lengths, $l_{\min }=$ $2 \pi / k_{\max }$, and $l_{\max }=2 \pi / k_{\min }$, together with the "favored" value $s=5 / 3$, one can write the corresponding energy loss rate as

$$
\begin{aligned}
\epsilon_{\text {isotropic }}= & \left(\frac{\pi}{2}\right)^{1 / 2}(\delta B)^{2} v_{\mathrm{e}} \\
& \times \frac{m_{\mathrm{e}}}{m_{\mathrm{i}}} l_{\max }^{-2 / 3} l_{\min }^{-1 / 3}\left[\ln \left(v_{\mathrm{e}} / V_{\mathrm{A}}\right)-\frac{1}{2}\right]
\end{aligned}
$$

where it has been assumed that $l_{\min } \ll l_{\max }$.

\subsection{Slightly anisotropic turbulence}

$$
(\Lambda=1-\eta,|\eta| \ll 1)
$$

In this case the integral (12) over $\mu$ can be written

$$
\begin{aligned}
& I\left(\mu_{\mathrm{L}}, \eta, s\right)=\int_{\mu_{\mathrm{L}}}^{1} \mathrm{~d} \mu \frac{\left(1-\mu^{2}\right)}{\mu\left[1-\eta\left(1-\mu^{2}\right)\right]^{1+(s / 2)}} \\
& \simeq \int_{\mu_{\mathrm{L}}}^{1} \mathrm{~d} \mu \frac{\left(1-\mu^{2}\right)}{\mu}\left[1+\left(1+\frac{s}{2}\right) \eta\left(1-\mu^{2}\right)\right] \\
& =-\left[1+\eta\left(1+\frac{s}{2}\right)\right] \ln \left(\mu_{\mathrm{L}}\right)-\frac{1}{2}\left[1+2 \eta\left(1+\frac{s}{2}\right)\right]\left(1-\mu_{\mathrm{L}}^{2}\right) \\
& \quad+\frac{1}{4}\left[1+\eta\left(1+\frac{s}{2}\right)\right]\left(1-\mu_{\mathrm{L}}^{4}\right)
\end{aligned}
$$

which, for $\mu_{\mathrm{L}} \ll 1$, is dominated by the logarithmic term, so that one has approximately

$I\left(\mu_{\mathrm{L}}, \eta, s\right) \simeq\left[1+\eta\left(1+\frac{s}{2}\right)\right] \ln \left(1 / \mu_{\mathrm{L}}\right)-\frac{1}{2}$

Also in this case, one can write

$(\delta B)^{2}=C_{\mathrm{B}}^{2} \int \mathrm{d}^{3} k\left[k_{\|}^{2}+k_{\perp}^{2}-\eta k_{\perp}^{2}\right]^{-(2+s) / 2}$

so that

$$
C_{\mathrm{B}}^{2}=\frac{(\delta B)^{2}}{4 \pi} \frac{s-1}{1-\frac{2}{3} \eta\left(1+\frac{s}{2}\right)} k_{\min }^{s-1}\left[1-\left(k_{\min } / k_{\max }\right)^{s-1}\right] .
$$

Combining Eqs. (11), (25) and (27), the energy loss rate in this case can be written in terms of the isotropic loss rate (Eq. (24)) as

$\epsilon=\left[1+\frac{2}{3}\left(1+\frac{s}{2}\right)(1-\Lambda)\right] \epsilon_{\text {isotropic }}$ 


\subsection{Highly anisotropic turbulence $(\Lambda \ll 1$ or $\Lambda \gg 1)$}

\subsection{1. $\Lambda \ll 1$}

This case corresponds most closely to the situation envisioned by Spangler (1991) of turbulence dominated by waves that closely verge on the direction of the ambient magnetic field. The integral (12) then has several limiting behaviours depending on the relative size of $\Lambda$ to $\mu_{\mathrm{L}}^{2}$, both of which are much less than unity. The factor $\mu^{2}+\Lambda\left(1-\mu^{2}\right)$ in Eq. (12) is dominated by $\mu^{2}$ except for the region $\mu \leq \Lambda^{1 / 2}$ when dominance is controlled by $\Lambda\left(1-\mu^{2}\right)$. So two limiting cases exist.

(i) $1 \gg \mu_{\mathrm{L}} \geq \Lambda^{1 / 2}$

In this situation, dominance of $\mu^{2}+\Lambda\left(1-\mu^{2}\right)$ is controlled by $\mu^{2}$ always. Then the integral (12) is approximated by

$$
\begin{aligned}
& I\left(\mu_{\mathrm{L}} \geq \Lambda^{1 / 2}, \Lambda \ll 1, s\right) \simeq \int_{\mu_{\mathrm{L}}}^{1} \mathrm{~d} \mu \frac{1-\mu^{2}}{\mu^{3+s}} \\
& =\frac{\mu_{\mathrm{L}}^{-(2+s)}-1}{2+s}-\frac{\mu_{\mathrm{L}}^{-s}-1}{s} \simeq \frac{\mu_{\mathrm{L}}^{-(2+s)}}{2+s} .
\end{aligned}
$$

The integral (17) for $\Lambda \ll 1$ can be approximated for arbitrary $s$ as

$$
\begin{aligned}
& J(\Lambda \ll 1) \simeq \int_{0}^{\Lambda^{1 / 2}} \frac{\mathrm{d} \mu}{\left[\Lambda\left(1-\mu^{2}\right)\right]^{(2+s) / 2}}+\int_{\Lambda^{1 / 2}}^{1} \frac{\mathrm{d} \mu}{\mu^{2+s}} \\
& \simeq \Lambda^{-(2+s) / 2}+(s+1)^{-1}\left[\Lambda^{-(2+s) / 2}-1\right] \simeq \frac{s+2}{s+1} \Lambda^{-\frac{s+2}{2}}
\end{aligned}
$$

The energy loss rate (20) can then be written

$$
\epsilon=\epsilon_{\text {isotropic }} \frac{s+1}{(s+2)^{2}}\left[\frac{\Lambda}{\mu_{\mathrm{L}}^{2}}\right]^{(2+s) / 2}\left[\ln \left(v_{\mathrm{e}} / V_{\mathrm{A}}\right)-\frac{1}{2}\right]^{-1}
$$

indicated that in this case the loss rate is reduced compared to the isotropic case by the factor

$$
\frac{s+1}{(s+2)^{2}}\left[\frac{\Lambda}{\mu_{\mathrm{L}}^{2}}\right]^{(2+s) / 2}\left[\ln \left(v_{\mathrm{e}} / V_{\mathrm{A}}\right)-\frac{1}{2}\right]^{-1} \ll 1 .
$$

(ii) $1 \gg \Lambda^{1 / 2} \geq \mu_{\mathrm{L}}$

For $\Lambda \ll 1$ we approximate $\Lambda+(1-\Lambda) \mu_{\mathrm{L}}^{2} \simeq \Lambda+\mu_{\mathrm{L}}^{2}$ so that in Eq. (15)

$$
\begin{aligned}
& { }_{2} F_{1}\left(1,1+\frac{s}{2} ; 2+\frac{s}{2} ; \frac{\Lambda}{\Lambda+(1-\Lambda) \mu_{\mathrm{L}}^{2}}\right) \simeq \\
& \frac{2+s}{2} \sum_{n=0}^{\infty} \frac{\Gamma\left(n+1+\frac{s}{2}\right)}{\Gamma\left(1+\frac{s}{2}\right) n !}\left[\psi(n+1)-\psi\left(n+1+\frac{s}{2}\right)\right. \\
& \left.\quad-\ln \left(\frac{\mu_{\mathrm{L}}^{2}}{\Lambda+\mu_{\mathrm{L}}^{2}}\right)\right]\left[\frac{\mu_{\mathrm{L}}^{2}}{\Lambda+\mu_{\mathrm{L}}^{2}}\right]^{n}
\end{aligned}
$$

and ${ }_{2} F_{1}\left(1,1+\frac{s}{2} ; 2+\frac{s}{2} ; \Lambda\right) \simeq 1$.

For $\Lambda \geq \mu_{\mathrm{L}}^{2}$ Eq. (32) further reduces to

$$
{ }_{2} F_{1}\left(1,1+\frac{s}{2} ; 2+\frac{s}{2} ; \frac{\Lambda}{\Lambda+(1-\Lambda) \mu_{\mathrm{L}}^{2}}\right) \simeq \frac{2+s}{2} \ln \left(\frac{\Lambda}{\mu_{\mathrm{L}}^{2}}\right)
$$

and the integral (12) is approximated by

$$
I\left(\mu_{\mathrm{L}} \leq \Lambda^{1 / 2}, \Lambda \ll 1, s\right) \simeq \frac{1}{2} \Lambda^{-(2+s) / 2} \ln \left(\frac{\Lambda}{\mu_{\mathrm{L}}^{2}}\right) .
$$

The energy loss rate can then be written

$$
\epsilon=\epsilon_{\text {isotropic }} \frac{1}{s+2} \ln \left(\Lambda / \mu_{\mathrm{L}}^{2}\right)\left[\ln \left(v_{\mathrm{e}} / V_{\mathrm{A}}\right)-\frac{1}{2}\right]^{-1}
$$

In this case the loss rate can be slightly enhanced (but by not more than a factor 2) compared to the isotropic case by the factor

$\ln \left(\Lambda / \mu_{\mathrm{L}}^{2}\right)\left[\ln \left(v_{\mathrm{e}} / V_{\mathrm{A}}\right)-\frac{1}{2}\right]^{-1} \simeq 2+\frac{\ln \Lambda}{\ln \left(v_{\mathrm{e}} / V_{\mathrm{A}}\right)}<2$.

\subsection{2. $\Lambda \gg 1$}

This case marries most readily to the Goldreich \& Sridhar (1995) argument that the plasma turbulence is dominated by long ribbon-like turbulence paralleling the magnetic field. Substituting $t=\mu^{2}$ the angular integral (12) can be approximated as

$$
\begin{aligned}
& 2 I=\int_{\mu_{\mathrm{L}}^{2}}^{1} \mathrm{~d} t \frac{1-t}{t}[t+\Lambda(1-t)]^{-(2+s) / 2} \\
& \simeq \Lambda^{-\frac{2+s}{2}} \int_{\mu_{\mathrm{L}}^{2}}^{\left(1+\Lambda^{-1}\right)^{-1}} \mathrm{~d} t \frac{1}{t(1-t)^{s / 2}} \\
& +\int_{\left(1+\Lambda^{-1}\right)^{-1}}^{1} \mathrm{~d} t \frac{1-t}{t^{(4+s) / 2}}
\end{aligned}
$$

The second integral is elementary so that for $\Lambda \gg 1$

$2 I \simeq \Lambda^{-(2+s) / 2} H+\frac{1}{2 \Lambda^{2}}$

where

$$
H=\int_{(1+\Lambda)^{-1}}^{1-\mu_{\mathrm{L}}^{2}} \frac{\mathrm{d} t}{t^{s / 2}(1-t)} .
$$

In Appendix A we show that for values of $s<2$ the integral (38) to lowest order in $\Lambda^{-1} \ll 1$ can be approximated by

$H \simeq-2 \ln \mu_{\mathrm{L}}$

yielding according to Eq. (37)

$I \simeq \Lambda^{-(2+s) / 2} \ln \mu_{\mathrm{L}}^{-1}$.

Correspondingly, starting from Eq. (17) and using the transformation formula

${ }_{2} F_{1}(a, b ; c ; z)=(1-z)^{c-a-b}{ }_{2} F_{1}(c-a, c-b ; c ; z)$

we obtain

$J(\Lambda)=\Lambda_{2}^{-1}{ }_{2} F_{1}\left(\frac{1-s}{2}, 1 ; \frac{3}{2} ; 1-\Lambda^{-1}\right)$ 
which for large $\Lambda \gg 1$ is well approximated by

$J(\Lambda) \simeq \Lambda^{-1}{ }_{2} F_{1}\left(\frac{1-s}{2}, 1 ; \frac{3}{2} ; 1\right)=\frac{1}{s \Lambda}$.

For the energy loss rate (20 in this case we then obtain)

$\epsilon \simeq \epsilon_{\text {isotropic }} \frac{s}{\Lambda^{s / 2}}$

which is reduced relative to the isotropic case by the large factor $\left(\Lambda^{s / 2} / s\right)$.

\subsection{Interlude}

The list of situations available is thus complete and we summarize our results in Table 1 . Note that $\mu_{\mathrm{L}}$ is given by the ratio of the Alfven and electron thermal speed and thus related to the instellar plasma beta $\beta=$ $B_{0}^{2} /\left(8 \pi n_{\mathrm{e}} k_{\mathrm{B}} T\right)$ as

$\mu_{\mathrm{L}}=\left(\frac{2 m_{\mathrm{e}} \beta}{m_{\mathrm{p}}}\right)^{1 / 2}=0.033 \beta^{1 / 2}$.

According to Table 1 we find the largest energy loss rates in the case of isotropic turbulence $(\Lambda=1)$ and about twice that loss rate for anisotropic turbulence with $\mu_{\mathrm{L}} \ll \Lambda \ll 1$. In the other anistropic cases the energy loss rates are much smaller than in the isotropic case with the relevant reduction factors given in Table 1 . In the following section we therefore calculate quantitative energy loss rates in the interstellar medium for the case of isotropic turbulence. With the help of Table 1 it is then straightforward to infer the energy loss rates for any anisotropic case.

\section{Results and conclusions}

\subsection{HII regions}

Spangler (1991) has argued that the wave damping is most appropriate for his "fluctiferous" media, taken to be described by HiI regions. In our numerical estimate of the isotropic energy loss rate we adopt exactly the same parameter values as given by Spangler (1991), thereby revealing most clearly the differences between our calculations and his values. According to Spangler the parameters of relevance are $l_{7}=1, L_{17}=1, s=5 / 3$, $v_{\mathrm{e}}=210^{7} \mathrm{~cm} \mathrm{~s}^{-1}$ (corresponding to a temperature of about $10^{4} \mathrm{~K}$ ), $V_{A}=10^{6} \mathrm{~cm} \mathrm{~s}^{-1}$ (so $v_{\mathrm{e}} \gg V_{A}$ as required for the Ginzburg damping formula to be valid), $v_{\mathrm{i}}=v_{\mathrm{e}} / 43$, and $(\delta B)=0.3 \mu \mathrm{G}$. According to Eq. (24) we obtain

$\epsilon_{\text {isotropic }}=6.6110^{-23} L_{17}^{-2 / 3} l_{7}^{-1 / 3} \quad \mathrm{erg} \mathrm{s}^{-1} \mathrm{~cm}^{-3}$.

If the outer scale length $l_{\max }$ is increased to $1 \mathrm{pc}\left(L_{17}=\right.$ 30) then $\epsilon_{\text {isotropic }}$ is reduced by a factor $30^{2 / 3}=9.65$, as are all other cases. If the magnetic field fluctuations are increased to $1 \mu \mathrm{G}$ from $0.3 \mu \mathrm{G}$ then also the energy loss rate (46) is increased by almost an order of magnitude.

The corresponding radiative cooling rate in this fluctiferous medium is given by Spangler (1991) as about
Table 1. Energy loss rates for different anisotropy parameters

\begin{tabular}{|l|l|}
\hline Anisotropy $\Lambda$ & $\epsilon / \epsilon_{\text {isotropic }}$ \\
\hline \hline$\Lambda=1$ & 1 \\
$\Lambda=1-\eta,|\eta| \ll 1$ & $1+\frac{2}{3}\left(1+\frac{s}{2}\right)(1-\Lambda)$ \\
$\Lambda \leq\left(V_{\mathrm{A}} / v_{\mathrm{e}}\right)^{2} \ll 1$ & {$\left[\frac{\Lambda v_{\mathrm{e}}^{2}}{V_{\mathrm{A}}^{2}}\right]^{\frac{2+s}{2}}\left[\ln \left(v_{\mathrm{e}} / V_{\mathrm{A}}\right)-\frac{1}{2}\right]^{-1} \ll 1$} \\
$\left(V_{\mathrm{A}} / v_{\mathrm{e}}\right)^{2} \leq \Lambda \ll 1$ & $\ln \left(\Lambda v_{\mathrm{e}}^{2} / V_{\mathrm{A}}^{2}\right)\left[\ln \left(v_{\mathrm{e}} / V_{\mathrm{A}}\right)-\frac{1}{2}\right]^{-1} \leq 2$ \\
$\Lambda \gg 1$ & $s \Lambda^{-s / 2} \ll 1$ \\
\hline
\end{tabular}

$10^{-23} n_{\mathrm{e}}^{2}$ erg $\mathrm{s}^{-1} \mathrm{~cm}^{-3}$ so that, all other factors being equal, there is little disparity between the wave energy loss rate and the radiative cooling rate. Indeed, given the uncertainties on the values of $(\delta B), l_{\max }$ and $l_{\min }$, it is remarkable that the rates are as close as they are. The factor 6.6 enhancement of the isotropic energy loss rate (46) can be accounted for easily either by

(i) a large anisotropy factor $\frac{3}{5} \Lambda^{5 / 6}=6.6$ corrresponding to $\Lambda=17.7$,

or

(ii) a small anisotropy factor $(400 \Lambda)^{11 / 6}=1 / 6.6$ corresponding to $\Lambda=10^{-3}$.

\subsection{Diffuse interstellar medium}

For the diffuse interstellar medium Minter \& Spangler (1997) give a larger value for $\delta B=0.9 \mu \mathrm{G}$, and also give the outer and inner scales, $L_{17}=30, l_{7}=8$, while all other turbulence parameters remain unchanged. The isotropic energy loss rate (46) then reduces slightly to

$\epsilon_{\text {isotropic }}=3.0810^{-23} \quad \mathrm{erg} \mathrm{s}^{-1} \mathrm{~cm}^{-3}$.

According to Minter \& Spangler (1997) the radiative cooling rate in the diffuse interstellar medium at temperatures of $10^{4} \mathrm{~K}$ is $L_{\mathrm{R}}=510^{-24} n_{\mathrm{e}}^{2} \mathrm{erg} \mathrm{s}^{-1} \mathrm{~cm}^{-3}$. With an electron density of $n_{\mathrm{e}}=0.08 \mathrm{~cm}^{-3}$ this implies typically $L_{\mathrm{R}}=310^{-26} \mathrm{erg} \mathrm{s}^{-1} \mathrm{~cm}^{-3}$. The factor of $10^{3}$ enhancement in the energy loss rate (47) can again be accounted for by either a large anisotropy factor $\Lambda=7400$ or a small anisotropy factor $\Lambda=610^{-5}$.

\subsection{Nature of interstellar turbulence}

The rather close agreement of our linear Landau damping heating rates with the radiative cooling rate in interstellar HII regions and in the diffuse interstellar medium modifies the result of Minter \& Spangler (1997) who, on the basis of the calculation of Spangler (1991), conclude that the heating rate by linear Landau damping exceeds, in all cases, the radiative cooling rate of the diffuse interstellar medium by $3-4$ orders of magnitude. Because we evaluated our heating rates with the exact damping formula and exact input turbulence wave spectra this modification results from the manner of computation of the energy loss rate (5) by Spangler (1991). The integrals must be correctly integrated over all propagation angles of the plasma waves; the final result cannot then depend anymore on the wave 
propagation angle, unlike Eq. (15) of Spangler (1991) and Eq. (8) of Minter \& Spangler (1997).

In particular, the conclusion of Minter \& Spangler (1997), that interstellar turbulence does not behave like an ensemble of obliquely propagating fast magnetosonic waves, is not valid. Quite the opposite is true: the close match of Landau damping heating rate and the interstellar radiative cooling rate strongly supports the argument that interstellar turbulence mostly consists of obliquely propagating compressive fast magnetosonic waves. However, the power spectrum of these waves is not isotropic in wavenumber space but has to be very anisotropic with $\Lambda \simeq 10^{-3}$ or $\Lambda \simeq 18$ in the fluctiferous (HII) medium and with $\Lambda \simeq 610^{-5}$ or $\Lambda \simeq 7000$ in the diffuse interstellar medium. This determination of highly anisotropic wave spectra is in accord with the theoretical arbuments advanced by Goldreich \& Sridhar (1995) who convincingly argued for ribbon-like turbulence roughly paralleling the ambient magnetic field.

\subsection{Summary}

Because of the ongoing efforts to obtain an understanding of energy production and loss rates in the interstellar medium, with plasma waves being considered an integral component, it is necessary to continually update wave energy loss rates in the light of improvements in observations and their interpretations.

When considering individual plasma wave types, two factors are of importance. First, the approximations and conditions under which the wave damping rates are produced must be honored to ensure that one does not step outside the domain of validity of the approximation. Second, when integrating over a wave spectrum due care and diligence must be given in performing the integrals as accurately as possible and not approximating too early in the development. Failure to take such care can lead to inappropriate wave energy loss rates which do not reflect correctly the exact expressions.

The numerical estimates produced for linear Landau damping, using values from Spangler (1991), would seem to indicate that there is little, if any, disparity between radiative cooling rates and wave energy loss rates for the fluctiferous ( $\mathrm{H} \mathrm{II}$ ) and the diffuse interstellar medium when the anisotropy in the wave power spectrum is properly accounted for. Our results indicate that the interstellar turbulence mostly consists of obliquely propagating compressive fast magnetosonic waves. However, the power spectrum of these waves is not isotropic in wavenumber space but has to be very anisotropic, either elongated highly along or perpendicular to the ordered magnetic field component.

Acknowledgements. We acknowledge partial support of this work by the Deutsche Forschungsgemeinschaft through Sonderforschungsbereich 191.

\section{Appendix A: Calculation of an integral}

The task is to calculate an approximation of the integral (38)

$H=\int_{(1+\Lambda)^{-1}}^{1-\mu_{\mathrm{L}}^{2}} \frac{\mathrm{d} t}{t^{s / 2}(1-t)}$

for values of $s<2$. Using Eq. (3.194) of Gradshteyn \& Ryzhik (1961) we may write $H$ as the difference of two hypergeometric functions

$$
\begin{aligned}
& H=\frac{2}{2-s}\left[\left(1-\mu_{\mathrm{L}}^{2}\right)^{(2-s) / 2}{ }_{2} F_{1}\left(1,1-\frac{s}{2} ; 2-\frac{s}{2} ; 1-\mu_{\mathrm{L}}^{2}\right)\right. \\
& \left.-(1+\Lambda)^{-(2-s) / 2}{ }_{2} F_{1}\left(1,1-\frac{s}{2} ; 2-\frac{s}{2} ;(1+\Lambda)^{-1}\right)\right] .
\end{aligned}
$$

According to Eq. (15.3.10) of Abramowitz \& Stegun (1972) we use

$$
\begin{aligned}
& { }_{2} F_{1}\left(1,1-\frac{s}{2} ; 2-\frac{s}{2} ; z\right)=\frac{2-s}{2} \sum_{n=0}^{\infty} \frac{\Gamma\left(n+1-\frac{s}{2}\right)}{\Gamma\left(1-\frac{s}{2}\right) n !} \\
& {\left[\psi(n+1)-\psi\left(n+1-\frac{s}{2}\right)-\ln (1-z)\right](1-z)^{n}}
\end{aligned}
$$

so that

$$
\begin{aligned}
H=( & \left.1-\mu_{\mathrm{L}}^{2}\right)^{(2-s) / 2}\left[\sum_{n=0}^{\infty} \frac{\Gamma\left(n+1-\frac{s}{2}\right)}{\Gamma\left(1-\frac{s}{2}\right) n !}[\psi(n+1)\right. \\
& \left.-\psi\left(n+1-\frac{s}{2}\right)-2 \ln \mu_{\mathrm{L}}\right] \mu_{\mathrm{L}}^{2 n} \\
& -(1+\Lambda)^{-(2-s) / 2} \sum_{n=0}^{\infty} \frac{\Gamma\left(n+1-\frac{s}{2}\right)}{\Gamma\left(1-\frac{s}{2}\right) n !} \\
& \times\left[\psi(n+1)-\psi\left(n+1-\frac{s}{2}\right)+\ln \left(1+\Lambda^{-1}\right)\right] \\
& \times\left(1+\Lambda^{-1}\right)^{-n}
\end{aligned}
$$

Because $\Lambda \gg 1$ we may neglect the second term in Eq. (51) with respect to the first term. Moreover, because $\mu_{\mathrm{L}} \ll 1$ the leading part of the remaining first sum is the $n=0$ term resulting in the approximation

$H \simeq-2 \ln \mu_{\mathrm{L}}$

\section{References}

Abramowitz, M., \& Stegun, I. A. 1972, Handbook of Mathematical Functions, National Bureau of Standards, Washington

Barnes, A. 1966, Phys. Fluids, 9, 1483

Cesarsky, C. J. 1980, ARA\&A, 18, 289

Cheng, K.-P., \& Bruhweiler, F. C. 1990, ApJ, 364, 573

Cox, D. P., \& Smith, B. W. 1974, ApJ, 189, L105

Ferriere, K. M., Zweibel, E. G., \& Shull, J. M. 1988, ApJ, 332, 984

Field, G. B., Goldsmith, D. W., \& Habing, H. J. 1969, ApJ, 155, L149 
Ginzburg, V. I. 1961, Propagation of Electromagnetic Waves in Plasma (Pergamon Press, New York)

Goldreich, P., \& Sridhar, S. 1995, ApJ, 438, 763

Gradshteyn, I. S., \& Ryzhik, I. M. 1965, Table of Integrals, Series, and Products (Academic Press, New York)

Hartquist, T. W., \& Snijders, M. A. J. 1982, Nature, 299, 783

Jenkins, E. B. 1978, Comm. Astrophys., 7, 121

Jokipii, J. R., \& Lerche, I. 1969, ApJ, 157, 1139

Jokipii, J. R., Lerche, I., \& Schommer, R. A. 1969, ApJ, 157, L119

Kulsrud, R. M., \& Pearce, W. P. 1969, ApJ, 156, 445
Landau, L. D., \& Lifschitz, E. M. 1981, Hydrodynamik (Akademie-Verlag, Berlin)

Lerche, I., \& Schlickeiser, R. 1982, MNRAS, 201, 1041

McCammon, D., \& Sanders, W. T. 1990, ARA\&A, 28, 657

McCray, R., \& Snow, T. P. 1979, ARA\&A, 17, 213

McIvor, I. 1977, MNRAS, 178, 85

McKee, C. F., \& Ostriker, J. P. 1977, ApJ, 21118, 148

Minter, A. H., \& Spangler, S. R. 1997, ApJ, 485, 182

Rickett, B. J. 1990, ARA\&A, 28, 561

Spangler, S. R. 1991, ApJ, 376, 540

Spitzer, L. 1956, ApJ, 124, 20 